$2 \%$, suggesting that double gloving maintained a barrier between the wearer and patient in four out of five cases in which the outer glove had been breached. This added protection is important as the subjects were unaware of half of the punctures until the operation was over. The greater vulnerability of the left hand, especially the index finger, has been observed previously in right handed surgeons.'

Acceptance of double gloving seems to depend on the person: some subjects in this study removed their outer gloves more frequently than others. Surgical staff will have to balance the enhanced safety of introducing a second barrier between themselves and the patients against possible discomfort or reduced sensitivity and dexterity.

We thank the medical and nursing staff of the department of clinical surgery for their willing participation in this study.

1 Hussain SA, Latif ABA, Choudhary AAAA. Risk to surgeons: a survey of associated injuries during operations. Br $\mathcal{X}$ Surg 1988;75:314-6.

2 Brough SA, Hunt TM, Barrie WW. Surgical glove perforations. Br J Surg 1988;75:317.

3 Church J, Sanderson P. Surgical glove punctures. J Hosp Infect 1980;1:84. 4 Skaug N. Micropunctures of rubber gloves used in oral surgery. Int 7 Oral Surg 1976;5:220-5.

(Accepted 27 May 1988)

\section{Tetanus immunisation state in a general practice population}

\author{
A M Dixon, J A Bibby
}

The Surgery, 16 Carr Lane, Shipley, West Yorkshire BD182JT

A M Dixon, MB, trainee general practitioner

J A Bibby, MRCGP, general practitioner

Correspondence to: $\mathrm{Dr}$ Bibby.

Tetanus immunisation state of respondents to survey

Tetanus is most common in adults, and the incidence rises with age.' At present the Department of Health and Social Security recommends that tetanus immunisation should consist of a primary course of three tetanus toxoid injections followed by a series of booster injections at intervals of five to 15 years. ${ }^{2}$ Provided a primary course has been given, even up to 30 years previously, a single booster is sufficient to restore protective levels of antibody. ${ }^{3}$ In this practice we have adopted a policy of giving tetanus boosters every five years.

Adults may have received primary immunisation in three different ways. Firstly, all people in the armed forces, including non-combatants, have been immunised since 1938 . Secondly, childhood immunisation became possible when diphtheria, tetanus, and pertussis vaccine was licensed in 1953; it became the policy of Bradford Health Authority in 1959" and national policy in $1961 .^{2}$ Thirdly, people may be immunised in casualty departments or by general practitioners or occupational physicians.

After one of our patients died of tetanus we investigated the immunisation state of the adult population in our practice.

\section{Patients, methods, and results}

We devised a questionnaire based on the three main ways of receiving primary tetanus immunisation. Respondents indicated their age and sex and whether they had served in the armed forces, received immunisation in infancy, or had a course of three tetanus

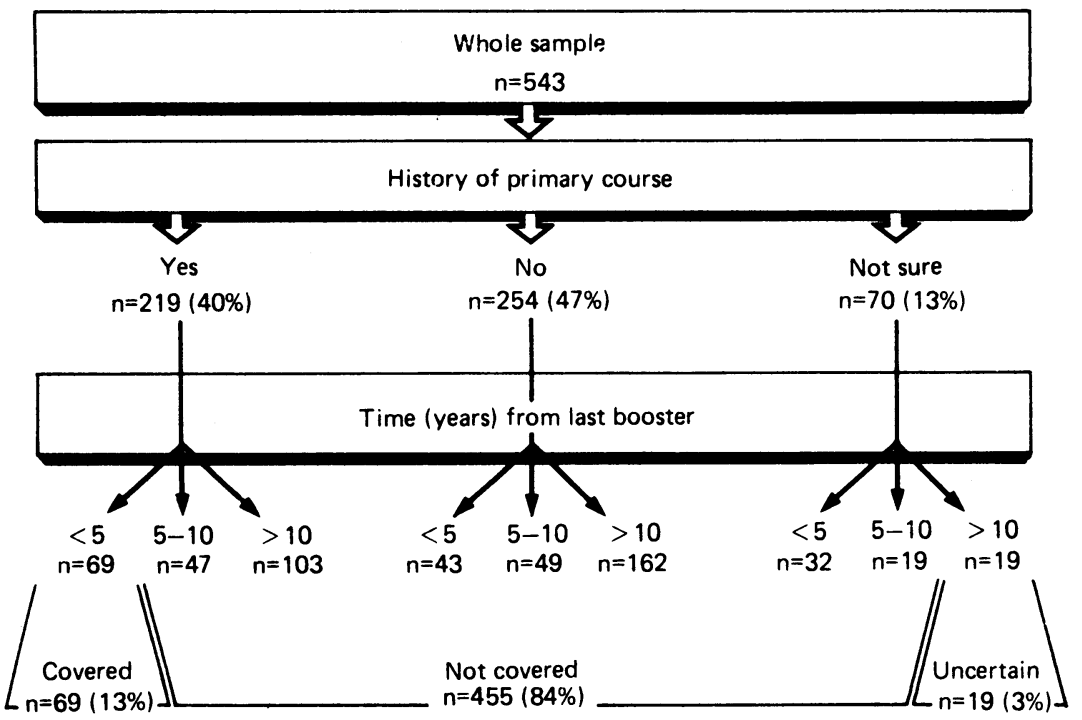

injections in adult life. We assumed that people born before 1958 were unlikely to have been immunised in infancy as they were over 1 year old by January 1959 . We also assumed that people who had received a primary course of immunisation as an adult would remember having attended for a series of three injections. The interval since their last tetanus injection was stated, and if this exceeded five years they were advised to attend for immunisation. Respondents indicated whether they planned to follow this advice.

Six hundred patients consecutively attending this surgery were given the questionnaire by the receptionist, and they completed it while waiting for their appointment. Of 543 who gave a valid response, only $69(13 \%)$ had received a primary course followed by a booster in the past five years; even when the criterion of a booster in the past 10 years was used only 116 were covered. Seventy patients, all born after January 1958 , were uncertain whether they had received primary immunisation as children. Of these, 19 had received a booster in the past five years and may therefore have been adequately covered. The remaining 455 (84\%) patients were not adequately covered (figure). The people with the lowest prevalence of immunisation were women aged $30-60$. Of these, only 26 out of 160 (16\%) had received a primary course compared with 193 of the $383(50 \%)$ other patients.

\section{Comment}

Although this was a limited study, the results suggested that the adult population of this practice was poorly immunised against tetanus. There are, however, no national statistics for comparison. The response to the advice to attend for immunisation was poor: of 412 respondents who had not received a tetanus injection in the past five years, only 177 intended to make an appointment, and one month later only 37 had done so. This rate of uptake might be improved by giving advice and a leaflet at a consultation or at a health screening clinic. In our practice over $90 \%$ of patients found at a health screening clinic to need a course of immunisation against tetanus completed it. A more workable approach might be to target efforts at the most poorly immunised group-that is, women aged 30-60. This could be done when cervical smears are carried out at five year intervals by reviewing the tetanus immunisation state and offering vaccination if necessary.

Public Health Laboratory Service Communicable Disease Surveillance Centre Tetanus surveillance: England and Wales, 1981-3. Br Med $\mathcal{J}$ 1985;290:696-7

2 Joint Committee on Vaccination and Immunisation. Immunisation against infectious disease. London: DHSS, Scottish Home and Health Department, and Welsh Office, 1988:25-29.

3 Simonsen O, Kjeldsen K, Heron I. Immunity against tetanus and effect of revaccination $25-30$ years after primary vaccination. Lancet 1984;ii: 1240-2. Boyd JSK. Tetanus in African and European theatres of war, 1935-45. Lance 1946;i:113-9.

5 Douglas J. The heulth of Bradford 1959. Annual report of the medical officer of health and principal school medical officer. Bradford: Bradford Corporation Health Department, 1960.

(Accepled 7 June 1988 ) 